\section{BMJ Open} Ophthalmology

\title{
Intraocular lens dislocation: a novel in- situ scleral refixation technique using a 25 Gauge trocar in the anterior chamber
}

\author{
Fabrizio Giansanti, ${ }^{1}$ Ruggero Tartaro, ${ }^{1}$ Tomaso Caporossi, ${ }^{1}$ Vittoria Murro, \\ Alfonso Savastano, ${ }^{1}$ Francesco Barca, ${ }^{1}$ Daniela Bacherini, ${ }^{1}$ Tito Fiore, ${ }^{2}$ \\ Carlo Cagini, ${ }^{2}$ Stanislao Rizzo ${ }^{1}$
}

To cite: Giansanti F, Tartaro R, Caporossi T, et al. Intraocular lens dislocation: a novel in-situ scleral refixation technique using a 25 Gauge trocar in the anterior chamber. BMJ Open Ophthalmology 2018;3:e000174. doi:10.1136/ bmjophth-2018-000174

- Additional material is published online only. To view please visit the journal online (http://dx.doi.org/10.1136/ bmjophth-2018-000174).

Received 22 April 2018 Accepted 2 October 2018

Check for updates

\section{(C) Author(s) (or their} employer(s)) 2018. Re-use permitted under CC BY-NC. No commercial re-use. See rights and permissions. Published by BMJ.

\section{${ }^{1}$ Dipartimento}

Neuromuscoloscheletrico e Organi di Sensi, Università degli Study di Firenze, Florence, Italy ${ }^{2}$ Division of Ophthalmology, Department of Surgery and Biomedical Science, University of Perugia, S Maria della Misericordia Hospital, Perugia, Italy

Correspondence to Ruggero Tartaro; ruggero. tartaro@gmail.com

\section{ABSTRACT}

Objective Intraocular lens (IOL) repositioning using a closed-eye approach could be carried out in some selected cases. Our study focuses on the efficacy and safety of a IOL closed-eye repositioning technique using scleral suture, which is performed using a trocar as an intrastromal limbal guide.

Methods and analysis Thirty-one eyes of 31 patients with late IOL dislocation operated on between January 2015 and May 2017 were included in this retrospective non-comparative consecutive case series study. The patients had a single-piece in-the-bag dislocation or a 3-pieces in-the-bag or out-of-the-bag dislocation. The patients underwent an anterior vitrectomy and a scleral refixation in a closed chamber using a 10/0 polypropylene suture passed through a 25 Gauge trocar inserted in the anterior chamber.

Results The mean follow-up time was 19.54 months. Average preoperative best-corrected visual acuity (BCVA) was 0.73 LogMar $( \pm 0.21 \mathrm{SD})$; while average postoperative BCVA was 0.27 LogMar ( \pm 0.23 SD). Fifteen patients underwent anterior pars plana vitrectomy (PPV) while 16 patients did not; moreover, two patients underwent PPV. Six patients had an increase of postoperative intraocular pressure, two patients had postoperative decentration, two patients had postoperative cystoid macular oedema, none of the patients had major complications such as retinal detachment, choroidal detachment, malignant glaucoma, irreversible corneal decompensation and endophthalmitis. Conclusion We can affirm that our technique may be safe and useful in the case of 3-piece in-the bag or out-of the bag dislocated IOLs and also in the case of in-the-bag single-piece dislocated IOLS.

\section{INTRODUCTION}

Intraocular lens (IOL) dislocations are challenging complications and may be spontaneous or associated with traumas; it is estimated that they occur in $1.7 \%$ of postcataract surgery patients after 25 years. ${ }^{1}$

IOL dislocation can be divided into in-the bag IOL dislocation and out-of-the bag IOL dislocation.

IOL dislocations during the early postoperative time occur because of an inadequate capsular bag or ciliary sulcus support and they

\section{Key messages}

What is already known about this subject?

- Intraocular lens repositioning using scleral sutures in a closed-eye approach could be carried out in some selected cases.

What are the new findings?

A 25 Gauge trocar could be used as an intrastromal guide when the polypropylene sutures are passed.

How might these results change the focus of research or clinical practice?

This new technique will reduce polypropylene friction during the scleral fixation procedure.

are usually out of-the-bag dislocations, while late IOL dislocations usually occur because of a zonular weakness and they are in- the-bag dislocations. $^{23}$

Pseudoexfoliation syndrome (PEX) is the most common risk factor for late IOL-capsular bag dislocation, ${ }^{4}$ and it accounts for over $50 \%$ of the cases of late IOL dislocations ${ }^{5}$ and is usually associated with the in-the-bag type.

Untreated cases could develop severe decrease of visual acuity because of complete IOL dislocation in the vitreous chamber, chronic cystoid macular oedema (CME), anterior uveitis or retinal detachment.

Surgery for IOL dislocation is often challenging and different techniques, which can generally be classified into open-eye and closed-eye procedures, have been described. ${ }^{6-14}$

In the case of dislocated IOLs, it is also possible to find an altered capsular support which is no longer usable for bag implantation and many surgeons decide to remove the previously implanted IOL and carry out a secondary implantation using anterior chamber IOLs, iris claw IOLs and scleral fixation IOLs (SFIOLs); other surgeons use instead the same dislocated IOL, suturing 


\section{SURGICAL OPTIONS ALGORITHM}

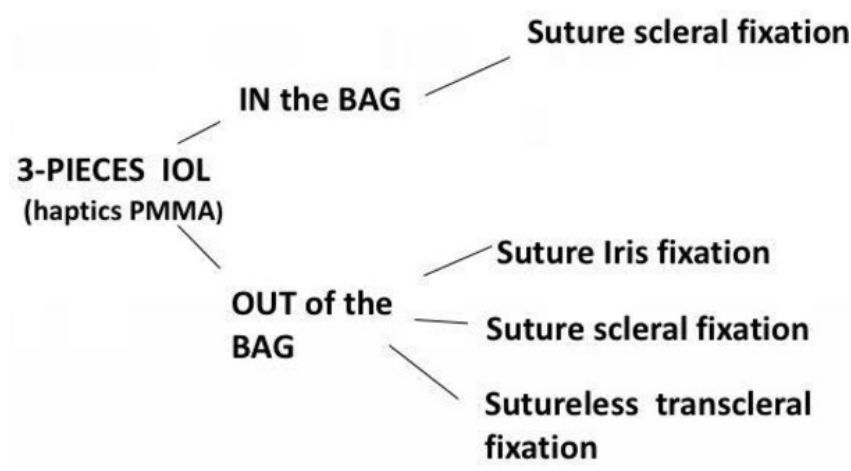

Figure 2 Surgical option algorithm in the case of 3-pieces IOLs. IOL, intraocular lens; PMMA, poly(methylmethacrylate).

it to the iris or fixing it with or without sutures to the sclera.

Substitution of a dislocated IOL involves an open eye approach through a large corneal incision which must be sutured, whereas the repositioning of a dislocated IOL using a closed-eye method is a better alternative; however, it presents greater surgical difficulty.

Our study focuses on the efficacy and safety of IOL, closed-eye, repositioning technique using scleral suture, which is performed using a trocar as an intrastromal guide.

\section{MATERIALS AND METHODS}

Thirty-one eyes of 31 patients with late IOL dislocation operated on between January 2015 and May 2017 were included in this retrospective non-comparative consecutive case series study. Institutional Review Board (IRB) / Ethics Committee approval was obtained.

All cases lacked sufficient capsule support to allow sulcus placement alone; furthermore, the patients in whom a single-piece, out-of-the bag dislocated IOL was found were excluded from the study, because the lens was extracted and a secondary implantation, using iris claw or SFIOL was performed.

In the case of a single-piece in-the bag dislocated IOL with capsular phimosis, the lens was extracted and a second implantation was carried out.

Our surgical flowchart in the case of single-piece or 3-piece IOL, in-the-bag or out-of-the-bag, dislocation is summarised in figures 1 and 2.

The data collected included demographic information, details on cataract extraction surgery, visual acuity, refraction, endothelial count, intraocular pressure,

Figure 1 Surgical option algorithm in the case of singlepiece IOLs. IOL, intraocular lens; PMMA, poly(methylmethacrylate). ocular biometry (measured using the IOLMaster 500, Carl Zeiss, Germany), information on fixation surgery, macular OCT examination, OCT assessment of the anterior segment and intraoperative and postoperative complications.

Unfortunately, preoperative refractive data were not available for all the patients because lens tilting and decentration avoid the measurements.

Postoperative spherical equivalents (SE) are summarised in table 1.

\section{Surgical technique}

Our technique used two double-armed 10-0 polypropylene (Prolene, Ethicon, USA) sutures to fix each IOL haptic to the sclera at each horizontal clock hour at a distance of $2 \mathrm{~mm}$ posterior to the limbus. All the surgical procedures were performed using retrobulbar block with ropivacaine $10 \%$ and lidocaine $2 \%$, mixed in equal volumes. Two conjunctival peritomies were conducted from 2 to 4 and from 7 to 10 clock hours and two radial relaxing incisions were created. Inferotemporal via pars plana trocar (Constellation, Alcon surgical, USA) was positioned and the infusion was opened; if vitreous was visualised in the anterior chamber two other via pars plana trocars at 2 and 10 o'clock were positioned and anterior vitreous vitrectomy (APPV) was performed (see online supplementary file 1 ).

On the other hand, if a vitreal bleeding was noticed, a pars plana vitrectomy (PPV) was conducted.

Two paracentesis, at 02:00 and 10:00 were performed and an ocular viscosurgical device (OVD) (Viscoat, Alcon surgical, USA) was injected into the anterior chamber. A 25-Gauge $(\mathrm{G})$ needle mounted on a $0.5 \mathrm{~mL}$ syringe was bent by $30^{\circ}$. Two limbal corneal paracentesis were carried out at 2 and 10 o'clock using a $15^{\circ}$ scalpel. A 25 G trocar was positioned through the 10 o'clock limbal paracentesis and the $25 \mathrm{G}$ needle was inserted through the sclera 2 mm from the limbus at 2 o'clock. A 10/0 Prolene straight double-armed needle was inserted at 10 o'clock through the trocar in the anterior chamber, under the IOL loop, docked inside the $25 \mathrm{G}$ needle and finally was conducted through the sclera and then out of the eye (see figure 3). A sterile white plastic plug was placed at the end of the needle to facilitate its visualisation out of the eye. The second needle of the second Prolene arm was conducted through the same trocar at 10 o'clock, and it was passed above the loop and then outside the eye docked in the same needle which was inserted at 2 o'clock $1 \mathrm{~mm}$ alongside the previous exit in order to create two SF points. The two extremities of Prolene were tensioned to fix the loop to the sclera. Equally, the other loop was fixed to the sclera using another double-armed Prolene 10/0 suture passed through a trocar positioned at the paracentesis at 2 o'clock and docked in a $25 \mathrm{G}$ needle at 10 o'clock, passed through the sclera $2 \mathrm{~mm}$ from limbus in two different $1 \mathrm{~mm}$-close holes, above and under the loop, in order to create two fixation points. The Prolene sutures were tensioned and knotted on both sides in order to fix 


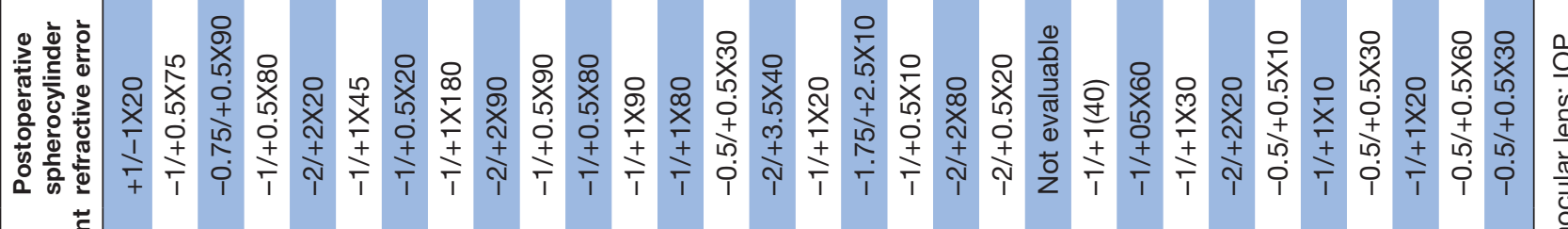

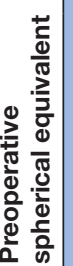

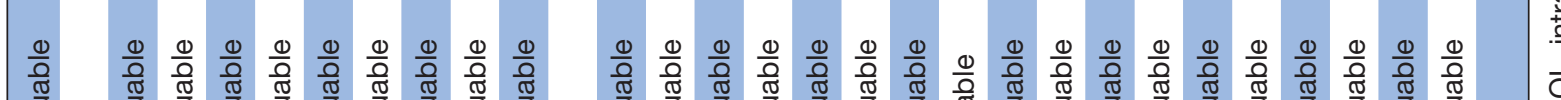

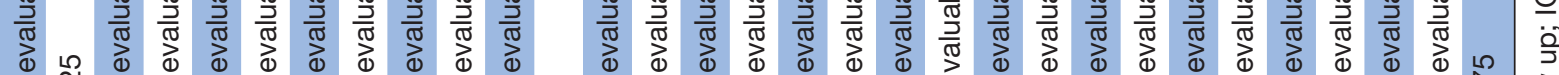

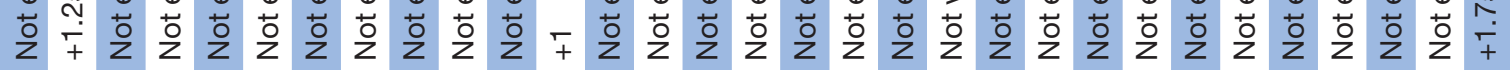

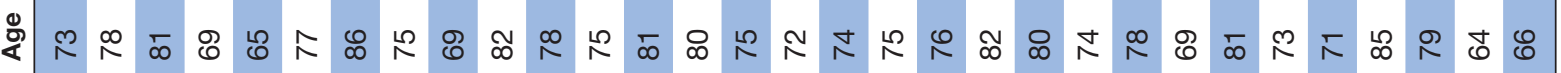

힌란

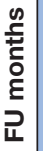

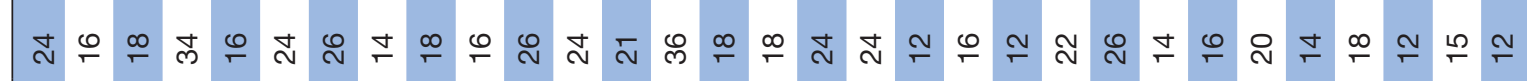

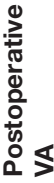

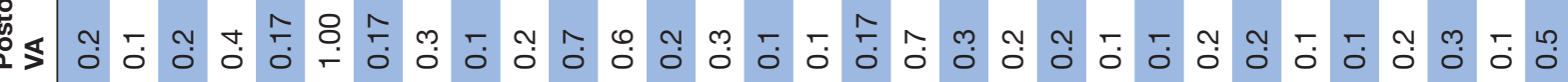

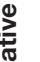

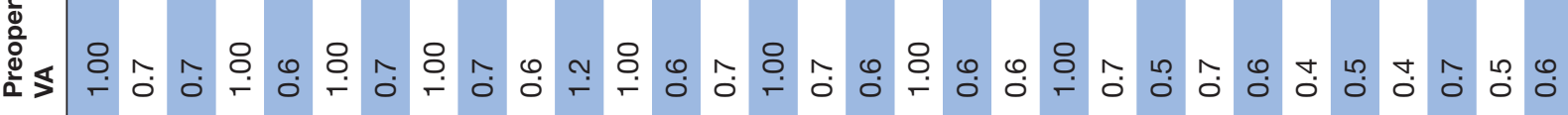

$\frac{0}{2}$

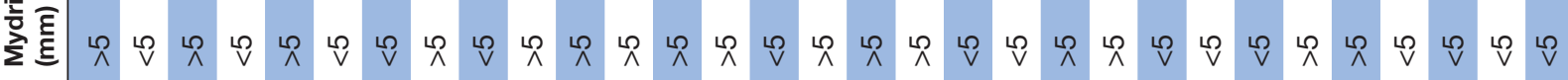

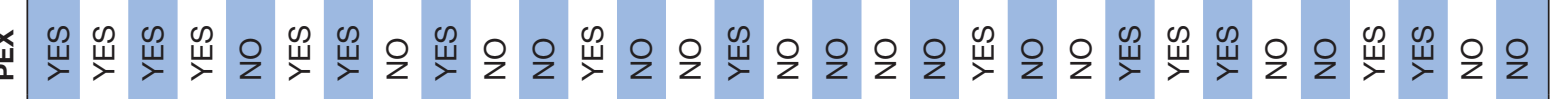

咅

范 한

$\frac{0}{0}$ के

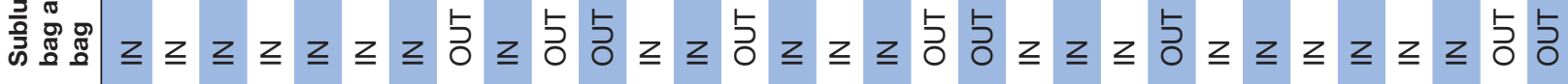

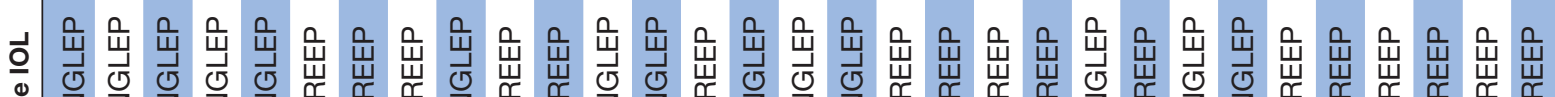

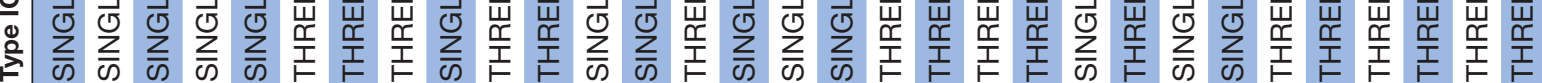




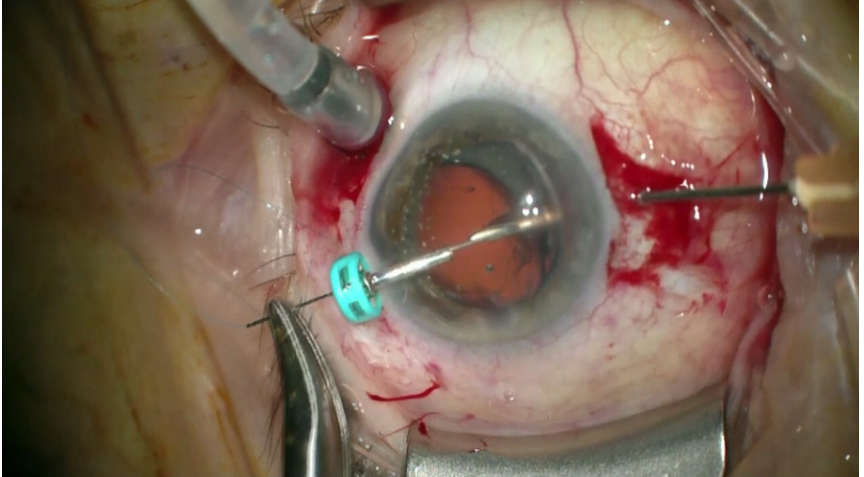

Figure 3 Intraoperative photograph showing the 10/0 polypropylene suture passed through a 25 Gauge trocar in the anterior chamber and docked inside a 25 Gauge needle 2 $\mathrm{mm}$ from the limbus.

and centre the IOL. The Prolene edges were cut $10 \mathrm{~mm}$ long and positioned under the conjunctiva and the tenon capsule in order to avoid conjunctival scratching and laceration (see figure 3). The OVD was removed from the anterior chamber; the anterior vitreous, if present, was cut and aspirated from the anterior chamber using the vitrectomy probe. Furthermore, the retina periphery was explored in order to avoid untreated rhegmatogenous lesions. Acetylcholine was injected in the anterior chamber to provoke miosis. The trocar was removed and a conjunctival absorbable suture, using vicryl $8 / 0$, was performed (see figure 4).

\section{RESULTS}

The average patient's age was 75.5 years. Seventeen of 31 patients had a 3-piece IOL, while 14 patients had a mono-piece IOL; 22 patients had in-the bag dislocations while 9 patients had out-of-the-bag dislocations. Fifteen of 31 patients had PEX and, moreover, 15 patients had a pupillary diameter $<5 \mathrm{~mm}$ in maximal mydriasis. Fifteen patients underwent APPV while 16 patients did not. The results are summarised in table 1.

The mean follow-up time was 19.54 months. Average preoperative best-corrected visual acuity (BCVA) was 0.73

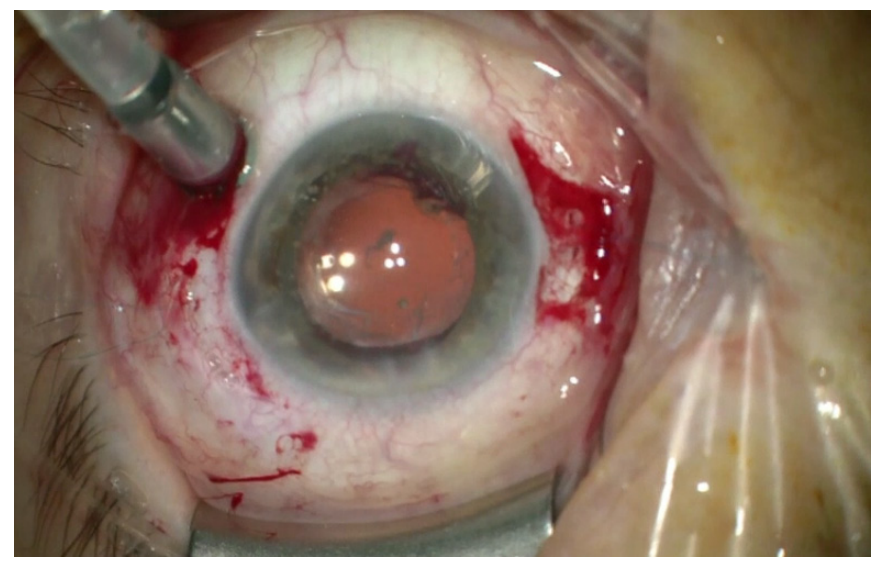

Figure 4 The refixated IOL at the end of the procedure. IOL, intraocular lens.

\begin{tabular}{ll}
\hline Table 2 & Postoperative complications \\
\hline Patient & Complication \\
\hline 1 & BLEEDING INTRAOP IN CA \\
2 & ELEVATED POSTOP INTRAOC PRESS \\
\hline 5 & BLEEDING INTRAOP IN CV \\
6 & EMC \\
\hline 10 & ELEVATED POSTOP INTRAOC PRESS \\
13 & DECENTRATION \\
\hline 14 & BLEEDING INTRAOP \\
15 & ELEVATED POSTOP INTRAOC PRESS \\
18 & BLEEDING INTRAOP \\
\hline 20 & ELEVATED POSTOP INTRAOC PRESS \\
21 & DECENTRATION ${ }^{\star}$ \\
22 & ELEVATED POSTOP INTRAOC PRESS \\
26 & ELEVATED POSTOP INTRAOC PRESS \\
\hline 29 & EMC \\
\hline
\end{tabular}

LogMAR $( \pm 0.21 \mathrm{SD})$; while average postoperative BCVA was $0.27 \operatorname{LogMAR}( \pm 0.23 \mathrm{SD})$.

Postoperative SE was -0.58 Dioptres (D) $( \pm 0.24 \mathrm{SD})$.

We have found a modest postoperative myopic SE in every case, as is to be expected from the more anterior position of the lens in relation to the original location.

Unfortunately, preoperative refractive data were not available for all the patients because lens tilting and decentration avoid the measurements, for which we cannot perform a statistical analysis to evaluate refractive changes.

Two patients had intraoperative anterior chamber bleeding, and in both cases, it resolved after a Viscoat injection and blood aspiration using the vitrectomy probe; two patients had vitreous chamber bleeding which was resolved by raising the infusion pressure to $50 \mathrm{~mm}$ $\mathrm{Hg}$ for $2 \mathrm{~min}$ and PPV.

Six patients had an increase of postoperative intraocular pressure which was resolved with medical therapy: of these patients, four had PEX (see table 2).

In one patient, the lens was found decentred downwards, and we decided to maintain his lens due to the acceptable vision arising from the free optic axis. Conversely, another patient decided to undergo VPP, IOL removal and secondary iris fixation IOL implantation.

In this patient, the postoperative IOL decentration caused the lens to move in order to have the edge along the visual axis and therefore the vision was compromised, and also refractive evaluation was impossible. After VPP+ secondary iris fixation IOL implantation, the patient was satisfied and postoperative complications such as CME did not arise. Two patients had postoperative CME which was resolved with nepafenac eye drops after 40 and 55 days, respectively; both of these patients had PEX and one underwent PPV during the SF procedure. 
None of the patients had major complications such as retinal detachment, choroidal detachment, malignant glaucoma, irreversible corneal decompensation and endophthalmitis.

\section{DISCUSSION}

Our study presents an IOL repositioning technique using scleral sutures.

The patients who had a single-piece, subluxated, out-of-the bag IOL were excluded from the study because the lens was extracted and a secondary implantation using iris claw or SFIOL was performed due to the risk of uveitis-glaucoma-hyphema syndrome. ${ }^{6}$ We have hypothesised that using this technique for single-piece out-of-the bag IOLs, which have stiff and thick haptics, could cause a continuous iris rubbing and inflammation which cause this syndrome. Uveitis-glaucoma-hyphema syndrome was not found in the follow-up of the patients who were included in the study.

We would like to empathise that our technique could be performed as well in 3-pieces luxated IOLs, and although the lens is refixated with two $1.5 \mathrm{~mm}$-from the limbus SF points, the poly(methyl-methacrylate) haptics did not cause excessive iris rubbing.

This fact is supported by the absence of pigment or inflammatory deposits on the lens plate.

Due to the fact that 15 of 31 patients had PEX, this study continues to consider this syndrome as the most common risk factor associated with late IOL-in-the-bag dislocation.

Fifteen patients underwent vitrectomy; 13 underwent only AVPP while a more complete vitrectomy combined with peripheral retinal observation and laser photocoagulation around retinal lattice degeneration, due to intraoperative vitreous chamber bleeding, was performed on two patients.

A dislocated IOL can be repositioned using a limbal or pars plana approach, the latter is the only technique available for IOLs entirely dislocated in the vitreous cavity. A benefit of this approach is the management of potential coexisting retinal complications; on the other hand, the limbal approach, with or without AVPP, may be sufficient in cases where the IOL is partially dislocated in the posterior chamber; and it avoids the complications of the pars plana entry, such as postoperative hypotony and endophthalmitis.

In a recent retrospective study, ${ }^{7}$ visual outcomes and complications of patients with SFIOLs and either anterior vitrectomy (AV) ( $n=36$ eyes) or posterior PPV $(n=47$ eyes) were evaluated. Visual acuity improvement was similar in both groups. Eyes in the SFIOL/AV group were more likely to experience lens dislocation (28\%) compared with those in the SFIOL/PPV group (9\%) $(p=0.036)$. Patients in the PPV group were more likely to experience a myopic shift and had a higher rate of IOL capture (23\%) compared with the AV group (3\%) $(\mathrm{p}=0.01)$. However, similarly to our study, this is a retrospective analysis and biases are inherent to this design. In our study, we decided to perform PPV only in two cases in whom vitreous chamber bleeding was detected intraoperatively. In our study, only two patients had postoperative decentration even though AVPP was carried out on 13 patients, and moreover, none of the patients had IOL capture.

Because of the fact that scleral flap creation is not performed and there is no IOL removal but only repositioning, our technique is carried out in no more than $30 \mathrm{~min}$.

Furthermore, it may cause less astigmatism because we perform the procedure in a closed chamber without carrying out the large corneal incisions, which are needed in the case of IOL substitution.

Moreover, the closed chamber approach maintained with a valved-trocar can help the surgeon due to the fact that we have less IOP fluctuations, caused by BSS and OVD exit.

The needles are inserted into the anterior chamber through a $25 \mathrm{G}$ trocar positioned inside a limbal corneal paracentesis; particular care must be taken when the needle is inserted, and it is useful to perform little backward and forward movements to ensure that you are inside the trocar lumen. If the needle plugs through the trocar plastic valves, it can be impossible to move the needle smoothly inside the eye and then to perform the operation; if this complication occurs it is sufficient to remove the trocar from the paracentesis, remove the plastic valves with two forceps and, then, free the needle; then the trocar could be reinserted, and the needle could be passed again through it. Furthermore, it is possible to use non-valved trocars but the anterior chamber could be less stable due to BSS leakage.

In the case of a 3-piece IOL, the anchor point is at the maximal loop concavity; on the other hand, in the case of a mono-piece IOL (eg, in SA60AT (Alcon, surgical) the anchorage is at the terminal button which, due to its shape, stops the Prolene suture from sliding.

It is important to knot the Prolene suture ends only after they are tensioned in order to control the IOL centring; in fact, centring regulation can be obtained only by tensioning and relaxing the Prolene along the horizontal meridian before the sutures are tied.

In the follow-up, we have not faced any clinically detectable lens tilting. The two SF points have created a good stability of the refixated IOL. Furthermore, both in in-the-bag and out-of the bag subluxations, the presence of the residual capsular bag in the first case or the posterior capsule in the second case increases IOL stability. Our technique could be used in luxated IOL as well, although after vitrectomy and capsular bag removal, we can risk more IOL tilting.

In the case of non-sufficient mydriasis, it is useful to use iridal retractors or a malyugin ring in order to visualise the IOL loop, due to the fact that it is preferable to not move the IOL inappropriately in order to avoid vitreous prolapse. 
We used Prolene sutures due to their non-absorbable properties; their duration and lysis time have been described in numerous papers. In a retrospective analysis of 63 eyes with SFIOLs affixed to the sclera with 10-0 polypropylene, two eyes (3\%) developed IOL dislocation secondary to suture breakage at 15 and 54 months postoperative time. ${ }^{8}$

Malta and colleagues ${ }^{9}$ reported a similar rate of suture breakage and IOL dislocation in a cohort of 105 eyes that underwent combined perforating keratoplasty with SFIOL implantation using 10-0 polypropylene sutures. They observed suture breakage in two eyes $(2 \%)$ at 5 and 8 years after the procedure; McAllister and colleagues reported five cases of Prolene breakage in eyes that underwent SFIOLs after 4.9 years. ${ }^{10}$

Buckley ${ }^{11}$ evaluated the use of $10-0$ polypropylene for SFIOLs in children; in his series, 4 out of 26 patients $(15 \%)$ experienced IOL dislocation secondary to suture breakage at a mean of 5.6 years following the initial surgery.

Pham-Duy and Hoder $^{12}$ examined five explanted 3-piece posterior chamber lenses using a scanning electron microscope to determine the changes. The intraocular time of the lenses varied from 1 week to 3 years. The polypropylene loops showed superficial cracks in all cases, but only in the curve and the insertion areas. The findings therefore indicated that the morphological changes are more probably caused by mechanical stress than by biological degradation alone.

We hypothesise that our technique, which reduces Prolene friction by the use of an anterior chamber trocar, avoiding the intrastromal suture incarceration, could reduce intraoperative suture wear and give better Prolene durability.

Using our technique, we did not have to create a scleral pocket, after the knot is made the Prolene edges are cut $10 \mathrm{~mm}$ long and are positioned under the Tenon capsule and the conjunctiva in order to avoid tissue erosion which could be observed in the case of short, sharp polypropylene ends. We have not found any sign of conjunctival erosion in our patients (see figure 5).

Conjunctival erosion, which can be observed in some series of SFIOLs, can increase the risk of endophthalmitis ${ }^{13-15}$ due to our suture placement technique, we did not encounter conjunctival erosion nor any case of endophthalmitis.

Moreover, we could perform our technique in eye with a thin sclera (such as myopic eyes) due to the fact that we do not carry out a scleral pocket.

Transcleral fixation of closed loop haptic acrylic posterior chamber IOL in aphakic non-vitrectomised eyes was reported by Agrawaal et al.; ${ }^{14}$ of 29 eyes of 24 patients treated using this technique, 2 patients had postoperative complications such as glaucoma and macular scarring. We had six patients who experienced postoperative ocular pressure elevation which was resolved with topical therapy without consequences; none of our patients had macular complications.

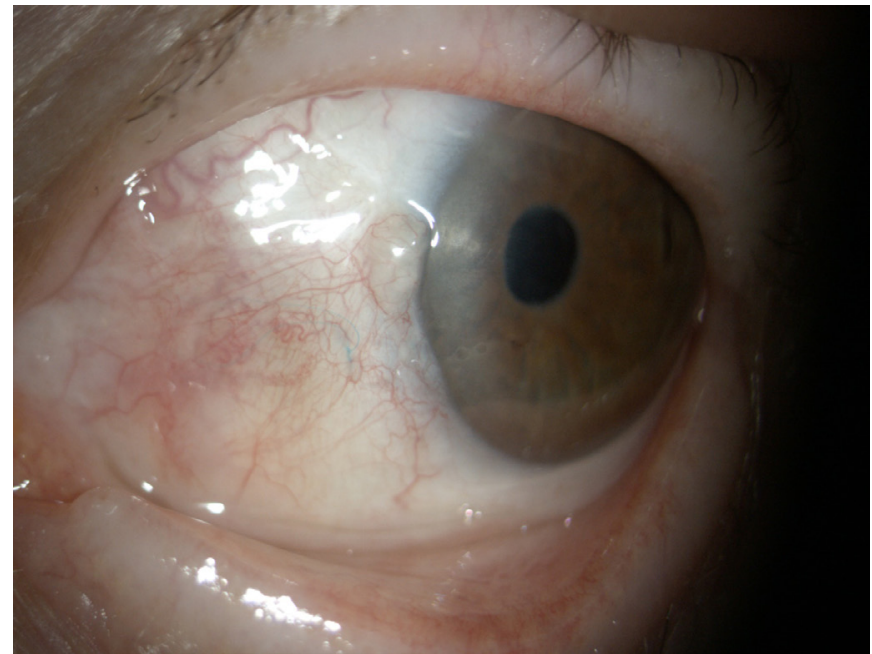

Figure 5 Anterior segment photograph showing the polypropylene suture under the conjunctiva after the operation.

Although CME is a common complication after IOL repositioning, mostly if iris suture is performed or if postoperative iris chafing is present, only two patients had postoperative CME which was resolved with nepafenac eye drops after 40 and 55 days, respectively; both of these patients had PEX and one underwent VPP during the SF procedure, due to intraoperative posterior bleeding.

Two patients had postoperative decentration, but only one of them had to perform a VPP+secondary IOL implantation because decentration affected the visual axis that was interrupted by the IOL edge.

Corneal endothelial failure which requires posterior lamellar keratoplasty, retinal detachment and choroidal detachment were not observed in our series while many authors describe these complications; for example, Luk and colleagues ${ }^{15}$ had to face these issues, even though in a larger series of patients (109 eyes).

McAllister and colleagues also experienced major complications which needed further interventions such as retinal detachment, uncontrolled glaucoma, corneal decompensation. ${ }^{10}$

\section{CONCLUSION}

To summarise we can affirm that our technique may be useful in the case of 3-piece in-the bag or out-of the bag dislocated IOLs and also in the case of in-the-bag singlepiece dislocated IOLs.

The technique is quick, safe and we did not find any postoperative major complications.

Moreover, we have found only two cases of IOL tilting in the follow-up.

We have found a modest myopic SE in every case, as is to be expected from the more anterior position of the lens in relation to the original location.

Furthermore, we suggest that using a trocar to access the anterior chamber with Prolene causes less stress to the suture in the intrastromal limbal passage and we may 
obtain more prolonged durability of the repositioned IOL in its correct centred position.

Our technique, leaving the ends of the Prolene long, under the conjunctiva and the Tenon capsule, makes it possible for us not to perform the scleral pockets. In our case study, we have not observed any signs of conjunctival erosion.

Contributors SR and FG planned the study. FB, TC, SR and FG performed the surgical procedures. RT, AS, VM, DB, CC and TF collected the data and wrote the manuscript.

Funding The authors have not declared a specific grant for this research from any funding agency in the public, commercial or not-for-profit sectors.

Disclaimer None of the authors has commercial associations that might pose a conflict of interest in connection with the submitted paper. All procedures followed were in accordance with the ethical standards of the responsible committee on human experimentation (institutional and national) and with the Helsinki Declaration of 1975, as revised in 2000. Informed consent was obtained from all patients for being included in the study.

Competing interests None declared.

Patient consent Not required.

Provenance and peer review Not commissioned; externally peer reviewed.

Open access This is an open access article distributed in accordance with the Creative Commons Attribution Non Commercial (CC BY-NC 4.0) license, which permits others to distribute, remix, adapt, build upon this work non-commercially, and license their derivative works on different terms, provided the original work is properly cited, appropriate credit is given, any changes made indicated, and the use is non-commercial. See: http://creativecommons.org/licenses/by-nc/4.0/

\section{REFERENCES}

1. Ascaso FJ, Huerva V, Grzybowski A. Epidemiology, etiology, and prevention of late IOL-capsular bag complex dislocation: review of the literature. $J$ Ophthalmol 2015;2015:1-7.
2. Lee HJ, Min SH, Kim TY. Bilateral spontaneous dislocation of intraocular lenses within the capsular bag in a retinitis pigmentosa patient. Korean J Ophthalmol 2004;18:52-7.

3. Seo MS, Kim CR, Nah HJ, et al. Management of posteriorly dislocated intraocular lens using pars plana vitrectomy. Korean $J$ Ophthalmol 2000;14:80-4.

4. Karger RA, Jeng SM, Johnson DH, et al. Estimated incidence of pseudoexfoliation syndrome and pseudoexfoliation glaucoma in Olmsted County, Minnesota. J Glaucoma 2003:12:193-7.

5. Ascaso FJ, Huerva V, Grzybowski A. Epidemiology, etiology, and prevention of late IOL-capsular bag complex dislocation: review of the literature. J Ophthalmol 2015;2015:805706-

6. Chang DF, Masket S, Miller KM, et al. Complications of sulcus placement of single-piece acrylic intraocular lenses: recommendations for backup IOL implantation following posterior capsule rupture. J Cataract Refract Surg 2009;35:1445-58.

7. Cho BJ, Yu HG. Surgical outcomes according to vitreous management after scleral fixation of posterior chamber intraocular lenses. Retina 2014;34:1977-84.

8. Bading G, Hillenkamp J, Sachs HG, et al. Long-term safety and functional outcome of combined pars plana vitrectomy and scleral-fixated sutured posterior chamber lens implantation. Am J Ophthalmol 2007;144:371-7.

9. Malta JB, Banitt M, Musch DC, et al. Long-term outcome of combined penetrating keratoplasty with scleral-sutured posterior chamber intraocular lens implantation. Cornea 2009;28:741-6.

10. McAllister AS, Hirst LW. Visual outcomes and complications of scleral-fixated posterior chamber intraocular lenses. J Cataract Refract Surg 2011:37:1263-9.

11. Buckley EG. Safety of transscleral-sutured intraocular lenses in children. J Aapos 2008;12:431-9.

12. Pham-Duy T, Hoder D. [Scanning electron microscopy findings in explanted posterior chamber lenses]. Fortschr Ophthalmol 1989;86:200-2.

13. Kang HM, Chung EJ. Late-onset Citrobacter koseri endophthalmitis with suture exposure after secondary intraocular lens implantation. Korean J Ophthalmol 2011;25:285-8.

14. Agrawal S, Singh V, Gupta SK, et al. Transscleral fixation of closed loop haptic acrylic posterior chamber intraocular lens in aphakic nonvitrectomized eyes. Indian J Ophtha/mol 2015;63:649-53

15. Luk AS, Young AL, Cheng LL. Long-term outcome of scleral-fixated intraocular lens implantation. Br J Ophthalmol 2013;97:1308-11. 